Institute of Health Policy, Management and Evaluation, University of Toronto, Canada

2 London School of Hygiene and Tropical Medicine, UK

3 Saw Swee Hock School of Public Health, Singapore

4 Johns Hopkins Bloomberg School of Public Health, USA

5 Dalla Lana School of Public Health, University of Toronto, Canada

6 Independent Panel for Pandemic Preparedness and Response Secretariat

7 School of Public Health, Boston University, USA

8 Faculty of Medical and Health Sciences, University of Auckland, New Zealand

Correspondence to: $\mathrm{V}$ Haldane v.haldane@mail.utoronto.ca Cite this as: $B M J$ 2021;375:e067510 http://dx.doi.org/10.1136/bmi-2021-067510 Published: 29 November 2021

COVID-19 PREPAREDNESS AND RESPONSE: IMPLICATIONS FOR FUTURE PANDEMICS

\title{
Strengthening the basics: public health responses to prevent the next pandemic
}

Victoria Haldane and colleagues argue that to make covid-19 the last pandemic, public health responses to outbreaks must be strengthened, starting with their most basic functions

Victoria Haldane, ${ }^{1}$ Anne-Sophie Jung, ${ }^{2}$ Chuan De Foo, ${ }^{3}$ Mathias Bonk, ${ }^{6}$ Margaret Jamieson, ${ }^{1}$ Shishi Wu, ${ }^{5}$ Monica Verma, ${ }^{3}$ Salma M Abdalla, ${ }^{6,7}$ Sudhvir Singh, ${ }^{6,8}$ Anders Nordström, ${ }^{6}$ Helena Legido-Quigley ${ }^{2,3}$ , 6

The covid-19 pandemic has challenged countries to mount and sustain comprehensive public health responses that identify and control an emerging infectious hazard. These challenges have persisted into 2021 and likely well beyond. Countries continue to grapple with the intersecting impact of ongoing outbreaks, inequitable vaccination, weak public health systems, inadequate social support, and variable political leadership. The unprecedented scale and duration of the pandemic have rightly raised questions as to what makes a robust and sustainable public health response. The answers to these questions become ever more pressing and complex as we enter the third year of the pandemic. Here, we reflect on three key questions: What are the basics of a public health response? How can these be strengthened for better pandemic preparedness and response? What is the way forward?

\section{What are the basics of a public health response?}

The basics of public health during infectious disease outbreaks aim to identify the pathogen and those exposed to it, monitor spread in the community, break chains of transmission, and communicate risk to the public. ${ }^{1}$ Public health activities, particularly those embedded within routine health service delivery, are crucial to identifying signals, such as unusual disease, that indicate an emerging outbreak. ${ }^{2}$ Once an unusual disease is detected and an outbreak confirmed, core public health functions operate to identify individuals and their contacts and break chains of transmission in communities.

Ongoing surveillance is foundational to understanding disease transmission and guide decision making. Comprehensive surveillance, including genomic surveillance, is reliant on accurate and timely testing to identify whether a person is, or has been, infected and to shed light on changes in the pathogen, drivers of infections, and their distribution across different populations. ${ }^{34}$ Contact tracing, both forwards to identify exposures and backwards to identify sources, is equally important in breaking chains of transmission. ${ }^{5}$ Once individuals are identified, quarantine restricts the movement of their close contacts and separates them from non-contacts to prevent new cases. Supporting contact quarantine and case isolation is often a weak link in public health functions, and social and financial supports should be in place to ensure people are able to complete their quarantine or isolation period.

These functions are interrelated and dependent on systems and infrastructures to ensure their maintenance and sustainability. For example, surveillance systems rely on laboratory systems to process diagnostic tests, the necessary skilled laboratory workforce, supplies and equipment to process tests, and mechanisms to communicate timely results. ${ }^{7}$ Core to such systems is a trained and protected health workforce able to reach even the most marginalised communities. Public health functions and systems are underpinned by public health and social measures, such as mask wearing, limits on gathering, and school or workplace closures, as well as social and financial support, such as paid sick days and debt relief, that encourage adherence. 89

The public health response to an emerging outbreak should be grounded in a protection of human rights with proactive and ongoing community engagement and risk communication. Individuals and community groups should be proactively involved in shared decision making to develop and sustain effective public health responses and accountability. ${ }^{10}$ Equally important is to recognise that public health - from response financing to the laboratories on the ground-is underpinned by geopolitics and global and local political decision making. These are crucial to the successes and failures of public health.

\section{How can we strengthen public health responses?}

Effective public health systems are those that strengthen the basics to be localised, responsive, integrated, and equitable (fig 1). 


\section{Public health surveillance, laboratory and referral systems}

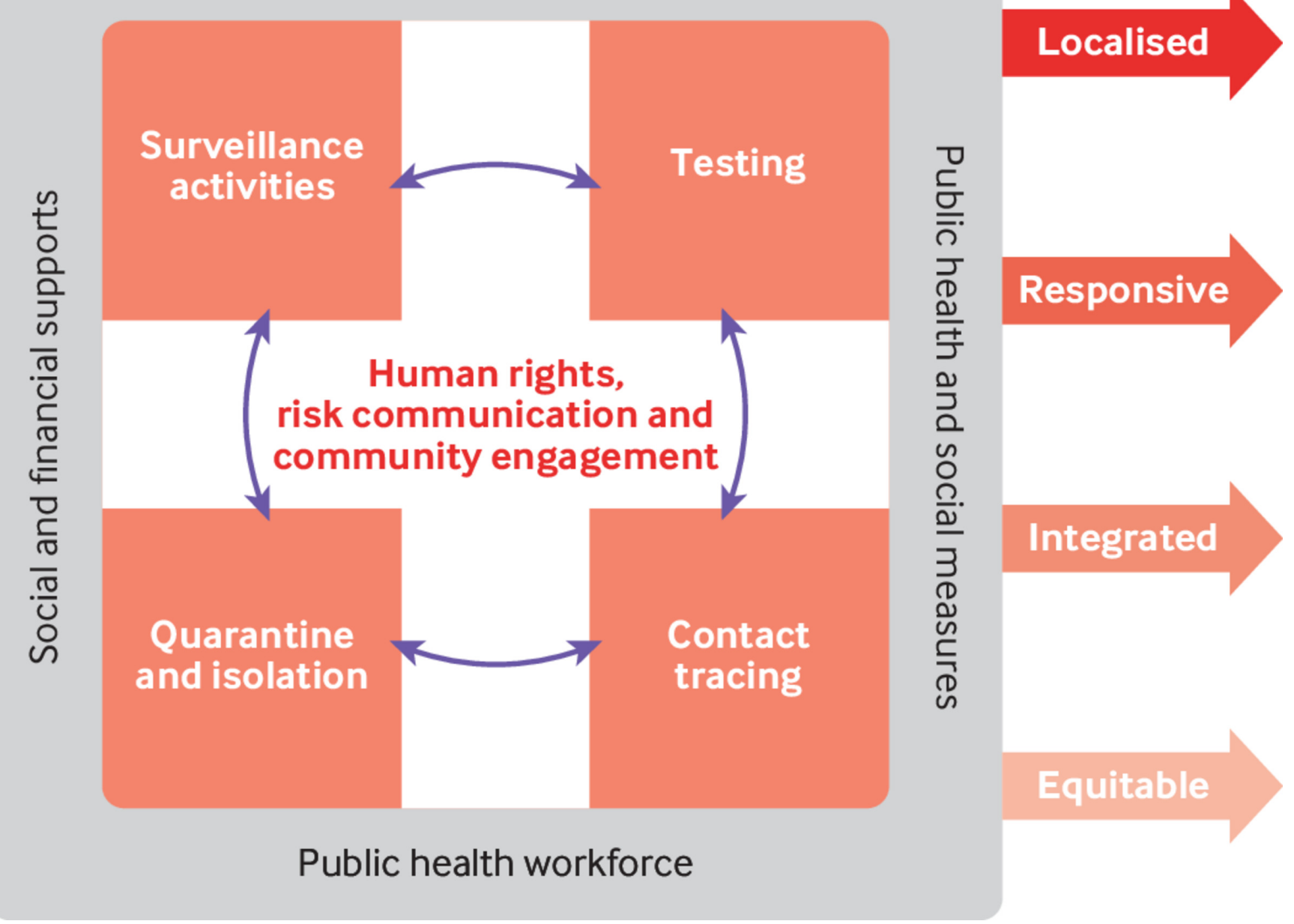

Fig 1 | Strengthening public health responses to infectious hazards

\section{Localised: engaging communities to develop and sustain responses}

Community engagement and local capacity building must be central to developing and implementing public health responses to outbreaks. ${ }^{11}$ Yet when considering the responses to covid-19, national efforts have often been disconnected from local needs, for both logistical and political reasons. Additionally, local public health efforts have failed many communities, hindered by inadequate financial and material support for pandemic preparedness. ${ }^{12}$ Countries can strengthen and tailor risk communication by building on existing community engaged programmes, such as community health workers or youth led programmes, and by monitoring and acting on public opinion and feedback. ${ }^{13}$ Yet deliberative spaces for community and civil society engagement must be sustainably incorporated through structures allowing for regular and routine consultation about the development, implementation, and evaluation of public health activities during emergencies. ${ }^{11}$

Creating spaces for community representation and civil society engagement is an essential basis for accountability in public health responses. Consideration must be given to equitable representation, whereby those who are often silenced or not given a voice because of their political status (eg, unregistered migrants) or societal status (eg, women, LGBTQ+) must be included and represented in debates. Consultation must take place without fear of stigmatisation or prosecution, which might prove challenging in many countries. Importantly, these spaces must be accessible, and governments must ensure communication channels are available. This includes, for example, counteracting language barriers when communicating with migrant groups. While involving the most marginalised in every aspect of pandemic response is key, representation is highly political and sensitive to misuse. Questions must be asked around how specific groups can be safely reached and represented.

\section{Responsive: supporting the public health workforce}

Localised capacity building must extend to the public health workforce to ensure a system is responsive to the evolving epidemiological situation. Frontline public health workers must be trained and retained before, during, and after emergencies. ${ }^{14}$ During the covid-19 pandemic, several countries, including the UK and US, have relied on insufficiently trained contract staff for contact tracing. Expanding health workforce capacities should be coupled with training to ensure high quality public health activities. Importantly, the public health workforce should reflect the communities in which they work, including a diversity of ethnic, linguistic, class, and 
gender representation. Longstanding challenges, including tokenisation, barriers to training, and limited mentorship, must be dealt with to capacity build emerging public health workers. ${ }^{15}$ The public health workforce must also collaborate to achieve response goals and reach all communities by leveraging on "task shifting," which has increased during the pandemic as those best placed to complete a task are the ones who do it. ${ }^{16}$

The public health workforce must be supported by systems that span healthcare, laboratory, surveillance, and social functions. Systems must be responsive enough to scale up and meet the demands of outbreaks while linking across sub-national regions and feeding national and global monitoring systems. Public health authorities and governments should conduct routine evaluations of their capacities. Many countries, however, missed the opportunity to prepare for public health emergencies, as highlighted by the lack of country engagement in the joint external evaluation as called for by the International Health Regulations (2005). ${ }^{17} 18$ Concerningly, in many countries recommendations for pandemic preparedness were not implemented before 2020 and remain inadequately responsive. ${ }^{19} 20$

\section{Integrated: supporting sustained responses}

Covid-19 has also highlighted the lack of integration between systems, which ultimately limits our ability to prevent cases from becoming outbreaks. Systems equipped to process diagnostic tests-laboratory networks staffed by a skilled laboratory workforce, supplied with reagents and equipment to process tests, and with mechanisms to communicate timely resultsare key to an integrated outbreak response and provide crucial information to understand the epidemiological situation. ${ }^{7}$ In response to covid-19, countries expanded surveillance through active approaches, leveraging existing systems for influenza or other pathogens, and legislated mandatory covid-19 case reporting to a national level health agency. Covid-19 has also seen digital technology used on an unprecedented scale for timely gathering and sharing of surveillance data to detect outbreaks, identify changes in epidemiological trends, and plan public health responses. ${ }^{21}$ Although in most countries covid-19 surveillance has largely been managed by the public health sector, intersectoral collaboration has spurred the use of data collected outside healthcare settings-for example, through apps or mobility data. Surveillance and health data collection are not unproblematic: ethical considerations must be given to understand what is being measured, who is rendered visible and invisible, and to what effect or consequence. ${ }^{22}$

Public health must also be supported by functioning and equitable supply chains and delivery systems. The first year of the pandemic highlighted how resource constraints, inequitable access to supplies, and corruption hinder crucial public health activities. ${ }^{23} 24$ Supply shortages disproportionately impacted low and middle income countries, which were further challenged by insufficient technical and digital infrastructures requiring rapid scale-up. ${ }^{25}$ In 2020, and now, countries of the Global South have been left unable to access supplies, including vaccines, relative to countries of the Global North. COVAX was hailed as the first step in the right direction to counteract some of these inequalities. However, instead of systematic change to tackle longstanding inequitable global distribution of medical technologies, COVAX has become a charitable project or even a smokescreen to distract from more substantial reform. ${ }^{26}$ Systems integration that overcomes inequities in word and deed must make a concerted effort to prioritise local needs, even in the most marginalised communities.
Equitable: centring on human rights and community engagement

Deepening inequities are a hallmark of the covid-19 pandemic, with public health functions and public policy efforts disproportionately impacting those most vulnerable. ${ }^{27}$ Public health responses must uphold privacy, equity, and human rights. National and global covid-19 responses have exposed the fault line between health interventions and health and wellbeing for all. ${ }^{28} 29$ Public health programmes have historically underserved, and in some cases harmed, those most vulnerable, and they continue to do so with little reparation. ${ }^{30}$ Covid-19 is a case in point, where the burden has fallen most heavily on already disadvantaged and vulnerable populations. Public health measures bear challenges within but also between countries: with the rapid scale-up of responses, especially digital health technologies and border closures, the human rights aspect that must be an integral part of all public health measures has often been an afterthought. ${ }^{31}$ Consequently, public health measures should prioritise both stopping transmission and mitigating the effects of the policies.

\section{What is the way forward?}

The challenges amplified by covid-19 have persisted into 2021 and will continue. New questions are being raised as a variety of vaccines, used in different regimens across populations, and unclear guidance on what public health measures apply to whom and in what circumstances converge to accelerate the need for effective public health. The recommendations we offer are based on our analysis of national responses during the first year of the pandemic, but are equally important as countries transition to endemicity and in countries that have lower vaccination rates or natural immunity (box 1)..$^{32}$

\section{Box 1: Recommendations towards comprehensive public health systems}

- Invest in the frontline public health workforce to be able to detect and rapidly respond to emerging outbreaks.

- Strengthen public health systems at the local level that can be rapidly scaled up when needed and are integrated with the health system and social services.

- Prioritise rapid deployment of core public health functions-surveillance, testing, contact tracing, and quarantine-and ensure equitable access to supplies.

- Ensure all public health efforts are developed, implemented, and evaluated in partnership with communities, including oppressed and marginalised groups.

- Uphold privacy, equity, and human rights in all public health activities.

We must invest in fast acting and scalable public health systems in communities. This requires a frontline public health workforce capable of rapid response to emerging outbreaks and able to scale up and integrate with health and social systems. To prevent emerging outbreaks from becoming pandemics, core public health functions must be prioritised for rapid deployment, able to surge and with adequate and equitable access to resources and supplies necessary to safely conduct public health work. Public health must actively engage with communities, including the most marginalised, to develop, implement, and evaluate interventions. For these actions to be meaningful, public health must acknowledge its carceral history and uphold privacy, equity, and human rights in all response efforts.

These recommendations are not new, and since its emergence SARS-CoV-2 has consistently laid bare our broken and underfunded public health systems. Widespread community transmission, strained healthcare systems, depleted health workforces, and the 
devastating global loss of life are the consequences of public health systems not able to fully execute core functions in detecting and responding to outbreaks. Longstanding political and financial neglect to build local public health capacity and meet local needs has left the world vulnerable to infectious hazards both known and unknown.

As we enter the third year of the pandemic, we know that leaders must strengthen the basics of public health. The work ahead is to ensure that political will supports the basics and realises localised, responsive, integrated, and equitable public health systems. Crucial to this is to understand how countries can be encouraged to commit to the set of recommendations necessary to safeguard health and wellbeing in communities before, during, and after pandemics.

\section{Key messages}

- The covid-19 response has been dominated by public policies to reduce transmission, underpinned by crucial public health functions including surveillance, testing, contact tracing, and quarantine.

- Public health responses to pandemics must be people centred, include core public health functions with effective systems, and have complementary public policies and social supports able to rapidly scale up.

- Public health must strengthen the basics and acknowledge that current failures are the result of broken and underfunded public health systems. We must redefine public health to be more than public policies, but a robust community led effort to detect and suppress emerging outbreaks.

Contributors and sources: This analysis was part of the work commissioned by the Independent Pane for Pandemic Preparedness and Response, which reviewed the national responses of 28 countries. Data used for the analysis were collected through literature review of peer reviewed papers, policy documents, public reports, and articles that examined national and sub-national policy response; semi-structured interviews with country experts; national government written submissions of selected countries about measures implemented to contain covid-19; and validation of country specific data by experts through written consultation and roundtable discussion The analysis is separate from the independent panel's final report and has been facilitated by the independent panel secretariat. The views expressed are those of the authors and do not represent the views of the Independent Panel for Pandemic Preparedness and Response. VH, A-SJ, and HL-Q conceived and designed the paper. VH, A-SJ, CDF, RN, SA, MJ, MMJT, SW, AC, MV, PS, SMT, and HL-Q collected the data. VH, A-SJ, CDF, SW, and $\mathrm{HL}-\mathrm{Q}$ analysed the data and drafted the manuscript with input from all authors. All authors contributed to revising the manuscript.

Competing interests: We have read and understood BMJ policy on declaration of interests and have no interests to declare. The secretariat of the Independent Panel for Pandemic Preparedness and Response is independent.

Provenance and peer review: Commissioned; externally peer reviewed.

This collection of articles was proposed by The Independent Panel for Pandemic Preparedness and Response. Open access fees were funded by WHO and Singapore's National Medical Research Council (NMRC/CG/C026/2017_NUHS). The BM/commissioned, peer reviewed, edited, and made the decision to publish these articles. Kamran Abbasi was the lead editor for The BMJ

Fisher DA, Carson GGOARN Steering Committee. Back to basics: the outbreak response pillars. Lancet 2020;396:598. doi: 10.1016/S0140-6736(20)31760-8 pmid: 32818438

2 Wynn A, Moore KM. Integration of primary health care and public health during a public health emergency. Am J Public Health2012;102:e9-12. doi: 10.2105/AJPH.2012.300957 pmid: 22994249

3 Manabe YC, Sharfstein JS, Armstrong K. The need for more and better testing for COVID-19. JAMA 2020;324:2153-4. doi: 10.1001/jama.2020.21694 pmid: 33185688

4 Robishaw JD, Alter SM, Solano JJ, etal. Genomic surveillance to combat COVID-19: challenges and opportunities. Lancet Microbe 2021;2:e481-4. doi: 10.1016/S2666-5247(21)00121-X pmid: 34337584

5 Endo A, Leclerc QJ, Knight GM, etalCentre for the Mathematical Modelling of Infectious Diseases COVID-19 Working Group. Implication of backward contact tracing in the presence of overdispersed transmission in COVID-19 outbreaks. Wellcome Open Res 2021;5:239. doi: 10.12688/wellcomeopenres.16344.3 pmid: 33154980

6 World Health Organization. Contact tracing in the context of COVID-19: Interim guidance. WHO, 2021.

7 Matthews Q, da Silva SJR, Norouzi M, Pena LJ, Pardee K. Adaptive, diverse and de-centralized diagnostics are key to the future of outbreak response. BMC Biol 2020;18:153. doi: 10.1186/s12915-020-00891-4 pmid: 33115440
8 Ali ST, Wang L, Lau EHY, etal. Serial interval of SARS-CoV-2 was shortened over time by nonpharmaceutical interventions. Science 2020;369:1106-9. doi: 10.1126/science.abc9004 pmid: 32694200

9 Enria D, Feng Z, Fretheim A, et al. Strengthening the evidence base for decisions on public health and social measures. Bull World Health Organ 2021;99(9):610-A. doi: 10.2471/BLT.21.287054.

10 World Health Organization. WHO community engagement framework for quality, people-centred and resilient health services. WHO, 2017.

11 Gilmore B, Ndejjo R, Tchetchia A, etal. Community engagement for COVID-19 prevention and control: a rapid evidence synthesis. BMJ Glob Health 2020;5:e003188. doi: 10.1136/bmigh-2020-003188 pmid: 33051285

12 Yamey G, Schäferhoff M, Aars OK, etal. Financing of international collective action for epidemic and pandemic preparedness. Lancet Glob Health 2017;5:e742-4. doi: 10.1016/S2214-109X(17)30203-6 pmid: 28528866

13 Abdalla SM, Koya S, Jamieson M, Verma M, Haldane V. Investing in trust and community resilience: lessons from the early months of the first digital pandemic. BMJ 2021; (Forthcoming).

14 Williams SG, Fontaine RE, Turcios Ruiz RM, Walke H, ljaz K, Baggett HC. One field epidemiologist per 200000 population: lessons learned from implementing a global public health workforce target. Health Secur 2020;18(S1):S113-8. doi: 10.1089/hs.2019.0119 pmid: 32004135

15 Wong BLH, Siepmann I, Chen TT, etal. Rebuilding to shape a better future: the role of young professionals in the public health workforce. Hum Resour Health 2021;19:82. doi: 10.1186/s12960-021-00627-7 pmid: 34256785

16 The Pan-European Commission on Health and Sustainable Development. Drawing light from the pandemic: A new strategy for health and sustainable development. WHO EURO, 2021.

17 World Health Organization. International health regulations (2005). 3rd ed. WHO, 2005.

18 Bell E, Tappero JW, ljaz K, etalCDC JEE Team and WHO Geneva JEE Secretariat. Joint external evaluation-development and scale-up of global multisectoral health capacity evaluation process. Emerg Infect Dis 2017;23. doi: 10.3201/eid2313.170949 pmid: 29155678

19 Gupta V, Kraemer JD, Katz R, etal. Analysis of results from the joint external evaluation: examining its strength and assessing for trends among participating countries. J Glob Health 2018;8:020416. doi: 10.7189/jogh.08.020416 pmid: 30410738

20 Talisuna A, Yahaya AA, Rajatonirina SC, etal. Joint external evaluation of the International Health Regulation (2005) capacities: current status and lessons learnt in the WHO African region. BMJ Glob Health 2019;4:e001312. doi: 10.1136/bmigh-2018-001312 pmid: 31798983

21 Jajosky RA, Groseclose SL. Evaluation of reporting timeliness of public health surveillance systems for infectious diseases. BMC Public Health 2004:4:29

doi: 10.1186/1471-2458-4-29 pmid: 15274746

22 World Health Organization. Considerations for strengthening legal frameworks for digital contact tracing and quarantine tools for COVID-19. WHO, 2021

23 Ranney ML, Griffeth V, Jha AK. Critical supply shortages-the need for ventilators and personal protective equipment during the covid-19 pandemic. N Engl / Med 2020;382:e41. doi: 10.1056/NEJMp2006141 pmid: 32212516

24 Abbasi K. Covid-19: politicisation, "corruption," and suppression of science. BM/2020;371:m4425 doi: 10.1136/bmj.m4425 pmid: 33187972

25 McMahon DE, Peters GA, Ivers LC, Freeman EE. Global resource shortages during COVID-19: Bad news for low-income countries. PLoS Negl Trop Dis 2020;14:e0008412. doi: 10.1371/journal.pntd.0008412 pmid: 32628664

26 Harman S, Erfani P, Goronga T, Hickel J, Morse M, Richardson T. Global vaccine equity demands reparative justice - not charity. BMJ Glob Health 2021;6:e006504doi: 10.1136/bmjgh-2021-006504.

27 Chiriboga D, Garay J, Buss P, Madrigal RS, Rispel LC. Health inequity during the COVID-19 pandemic: a cry for ethical global leadership. Lancet 2020;395:1690-1. doi: 10.1016/S0140-6736(20)31145-4 pmid: 32419711

28 Allen-Scott LK, Hatfield JM, McIntyre L. A scoping review of unintended harm associated with public health interventions: towards a typology and an understanding of underlying factors. Int J Public Health 2014;59:3-14. doi: 10.1007/s00038-013-0526-6 pmid: 24381979

29 Gugglberger L. Can health promotion also do harm? Health Promot Int 2018;33:557-60. doi: 10.1093/heapro/day060 pmid: 30239777

30 Addiss DG, Amon JJ. Apology and unintended harm in global health. Health Hum Rights 2019;21:19-32.pmid: 31239611

31 Gasser U, lenca M, Scheibner J, Sleigh J, Vayena E. Digital tools against COVID-19: taxonomy, ethical challenges, and navigation aid. Lancet Digit Health 2020;2:e425-34. doi: 10.1016/S2589-7500(20)30137-0 pmid: 32835200

32 De Foo C, Grépin KA, Cook AR, etal. Navigating from SARS-CoV-2 elimination to endemicity in Australia, Hong Kong, New Zealand, and Singapore. Lancet 2021;398:1547-51. doi: 10.1016/S0140-6736(21)02186-3 pmid: 34619099

This is an Open Access article distributed under the terms of the Creative Commons Attribution IGO License (https://creativecommons.org/licenses/by-nc/3.0/igo/), which permits use, distribution, and reproduction for non-commercial purposes in any medium, provided the original work is properly cited. 